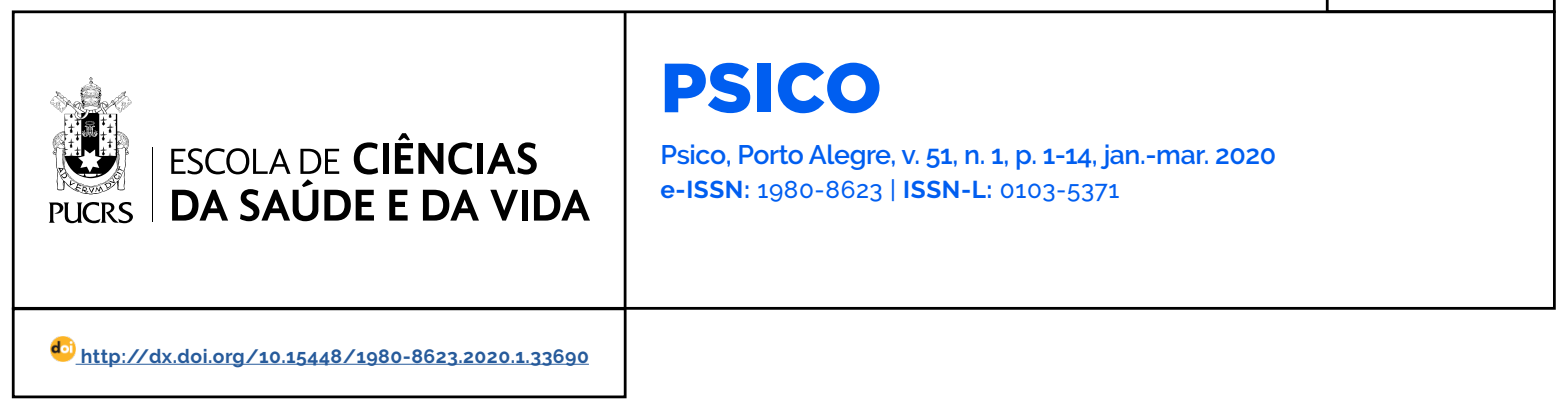

REVISÃO

\title{
Controle percebido em cuidadores de idosos no contexto familiar: variáveis associadas
}

\author{
Perceived control in caregivers of the elderly in the family context: associated variables \\ Control percibido en cuidadores de ancianos en el contexto familiar: variables asociadas
}

\section{Larissa Centofanti Lemos $^{1}$ \\ orcid.org/0000-0001-9350-394X larissacentofanti@gmail.com}

\section{Samila Sathler Tavares \\ Batistoni ${ }^{2}$}

orcid.org/0000-0002-8587-8298 samilabatistoni@gmail.com

\author{
Meire Cachioni \\ orcid.org/0000-0001-5220-410X \\ meirec@usp.br
}

\section{Anita Liberalesso Neri ${ }^{1}$ orcid.org/0000-0002-6833-7668 anitalbn@uol.com.br}

Enviado em: 5 abr. 2019. Aprovado em: 8 out. 2019. Publicado em: xx Xxx. 2020.

\section{(c) (1)}

Artigo está licenciado sob forma de uma licença Creative Commons Atribuição 4.0 Internacional.
Resumo: O presente estudo teve como objetivos testar um modelo explicativo do senso de controle de idosos cuidadores de idosos e comparar a frequência de respostas em senso de controle considerando respostas da amostra em fragilidade, sobrecarga, religiosidade, gênero e idade. Participaram 148 idosos cuidadores, sendo $77 \%$ mulheres e idade média de 70 anos. Senso de controle (Escala CASP-19), fragilidade autorreferida, sobrecarga (Escala de Sobrecarga de Zarit) e religiosidade (Índice de Religiosidade de DUKE) foram avaliados. O modelo de path analysis mostrou que o aumento do senso de controle se associou ao aumento da idade ( 6 $=0,209)$ por meio do aumento da religiosidade organizacional $(B=0,206)$ e, também, à diminuição da sobrecarga $(B=-0,375)$. O teste qui-quadrado revelou que os idosos que reportaram baixa sobrecarga $(p<0,001)$ e alta religiosidade organizacional ( $p$ $=0,002$ ) apresentaram alto senso de controle. Os dados evidenciaram que idosos cuidadores mais velhos que frequentavam encontros religiosos e que se percebiam menos sobrecarregados pela situação do cuidado tiveram maior senso de controle. Palavras-chave: cuidadores, idoso, crenças religiosas, adaptação psicológica.

Abstract: The present study had as objectives to test an explanatory model of the sense of control of elderly caregivers of the elderly and to compare the frequency of responses in sense of control considering the sample responses in frailty, burden, religiosity, gender and age. A total of 148 elderly caregivers participated, being $77 \%$ women and average age of 70 years. Sense of control (Scale CASP-19), frailty, burden (Zarit Burden Interview) and religiosity (DUKE Religious Index) were evaluated. The path analysis model showed that increased sense of control was associated with increasing age $(B=0.209)$ through increased organizational religiosity $(B=0.206)$, and was also associated with decreased burden $(\theta=-0.375)$. The chi-square test showed that the elderly who reported low burden $(p<0.001)$ and high organizational religiosity $(p=0.002)$ presented high sense of control. Data revealed that oldest elderly caregivers who attended religious meetings and who perceived themselves less burdened by the care situation had a greater sense of control.

Keywords: caregivers, elderly, religion, adaptation, psychological.

Resumen: Este estudio tuvo como objetivos probar un modelo explicativo del sentido de control de ancianos cuidadores de ancianos y comparar la frecuencia de respuestas en sentido de control considerando respuestas en fragilidad, sobrecarga, religiosidad, género y edad. Participaron 148 ancianos cuidadores, siendo 77\% mujeres y edad media de 70 años. El sentido de control (Escala CASP-19), fragilidad, sobrecarga (Escala de Sobrecarga de Zarit) y religiosidad (Índice de Religiosidad de DUKE) fueron evaluados. El modelo path analysis mostró que el aumento del sentido de control se asoció al aumento de la edad $(B=0.209)$ a través del aumento de la religiosidad organizacional $(B=0.206)$, y también se asoció a la disminución de la sobrecarga $(B=-0,375)$. La prueba de chi-cuadrado mostró que los ancianos que reportaron baja sobrecarga $(p<0.001)$ y alta religiosidad organizacional $(p=$ 0.002) presentaron alto sentido de control. Los datos evidenciaron que ancianos cuidadores mayores que frecuentaban encuentros religiosos y que se percibian menos sobrecargados por la situación del cuidado tuvieron mayor sentido de control. Palabras-clave: cuidadores, persona mayor, religión, adaptación psicológica. 
O senso de controle é considerado importante determinante do ajustamento e do bem-estar psicológico na velhice (Hyde, Wiggins, Higgs, \& Blane, 2003). Esse constructo psicológico é definido como um conjunto de crenças pessoais a respeito da própria capacidade pessoal em intervir de forma ativa no ambiente circundante, resultantes das experiências positivas e negativas do indivíduo ao interagir com o ambiente (Neri et al., 2018).

Certas condições do cuidado podem afetar o senso de controle do idoso cuidador, tais como: ser cônjuge do idoso alvo de cuidados e morar no mesmo domicilio o que leva a mais horas dispensadas para o cuidado e menos tempo de descanso; elevado período de tempo exercendo o papel de cuidador; auxilio em diversas atividades diárias e manejo de finanças; e, finalmente, haver menos contato e apoio social de amigos e outros familiares (Rafnsson, Shankar, \& Steptoe, 2015). Os autores referem também que tais condições podem causar dificuldade em manter as tarefas do cuidado, maior estresse e diminuição do bem-estar psicológico (Rafnsson et al., 2015). Dessa forma, o Modelo do Processo de Estresse de Pearlin e colaboradores, utilizado para a compreensão do fenômeno do cuidado e de seus desfechos em saúde mental do cuidador (Pearlin, Mullan, Semple, \& Skaff, 1990), permite auxiliar no vislumbre de particularidades quando aplicado entre cuidadores idosos.

Entre idosos cuidadores em contexto de vulnerabilidade social, a presença de comorbidades pode influenciar a sua capacidade funcional para exercer o cuidado (Santos-Orlandi et al., 2017a), e quando apresentam um quadro de fragilidade, a qualidade do cuidado prestado pode ser afetada, devido à sobreposição dos processos normais e patológicos associados ao envelhecimento e dos efeitos do ônus físico, psicológico e social ocasionado pelas tarefas de cuidado (Bianchi, 2015). O senso de controle é afetado pela ocorrência de doenças crônicas que prejudicam o funcionamento físico pleno do individuo (Sexton, King-Kallimanis, Layte, \& Hickey, 2015), o que também pode ocorrer no contexto de idosos cuidadores que apresentem sinais de fragilidade física.
As demandas objetivas do cuidado, dentre as quais estão os niveis de declínio funcional e cognitivo do idoso alvo de cuidados, podem ocasionar aumento da percepção de sobrecarga no cuidador (Jones et al., 2017; Oldenkamp, Hagedoorn, Wittek, Stolk, \& Smidt, 2017). A experiência de sobrecarga subjetiva, oriunda do exercício objetivo do cuidado, pode desafiar o senso de controle do cuidador, assim como o seu senso de autonomia e a visão geral da vida, aspectos ligados ao bem-estar psicológico de cuidadores idosos (Rafnsson et al., 2015).

Entretanto, estratégias de enfrentamento e recursos pessoais frequentemente utilizados na velhice, como a religiosidade (Koenig, 2017), são importantes variáveis considerando o panorama do Modelo de Pearlin sobre estresse do cuidador. Práticas religiosas públicas e privadas tendem a associar-se à diminuição do estresse percebido e da sobrecarga do cuidado, trazendo benefícios psicológicos ao cuidador, como melhor adaptação ao contexto do cuidado (Koenig, Nelson, Show, Saxena, \& Cohen, 2016).

As evidências de relações entre religiosidade e senso de controle demonstram a existência de construtos psicológicos, tais como o controle mediado por Deus, quando o indivíduo crê que compartilha o poder de ação sobre o ambiente com um ente superior (Krause, 2007). No cenário do envelhecimento, esse compartilhamento do controle com Deus auxilia na superação de problemas e situações dificeis e está relacionado à maior probabilidade de criar novos papéis sociais na velhice, os quais trazem prazer ao idoso, motivação, melhora na autoestima e novos significados de vida, além de manter seu bem-estar subjetivo (Krause \& Hayward, 2014), aspectos que podem auxiliar no exercício do cuidado a outro idoso.

Nesse sentido, o presente estudo tem como justificativa o fato de que os cuidadores idosos apresentam peculiaridades que os diferenciam dos cuidadores adultos, tais como as condições de saúde que apresentam, a forma como vivenciam os desafios e o estresse advindo do cuidado e de seus recursos pessoais para lidar com as dificuldades desse contexto e manter o bem-estar psicológico. No Brasil, o estudo do bem-estar 
subjetivo dos cuidadores idosos que cuidam de outro idoso no domicílio ainda é escasso.

Considerando o Modelo clássico de Pearlin e colaboradores (1990; Pearlin, 2009) sobre o estresse do cuidador, o presente estudo teve por objetivo testar um modelo de associações do senso de controle pessoal de idosos cuidadores de idosos no contexto domiciliar e verificar se há associações estatisticamente significativas do senso de controle com variáveis relativas aos niveis de fragilidade, aos níveis de sobrecarga percebida, aos niveis de religiosidade e ao gênero e à idade apresentados pelos idosos cuidadores. O presente estudo buscou, ainda, comparar a proporção de respostas da amostra em cada nivel de senso de controle de acordo com niveis de fragilidade, niveis de sobrecarga percebida, niveis de religiosidade e, finalmente, o gênero e a idade dos idosos cuidadores.

\section{Método}

\section{Participantes}

Os dados são provenientes de um banco construido com registros de entrevistas realizadas com cuidadores familiares de 60 anos e mais, no contexto de um estudo sobre o bem-estar psicológico de cuidadores idosos, conduzido nas cidades de Jundiai (38,5\%), Indaiatuba (29,1\%), Campinas (18,2\%) e Vinhedo (14,2\%), no estado de São Paulo. Foi constituída uma amostra de conveniência com 148 idosos cuidadores, a partir de recrutamento em clínicas privadas de Geriatria e de outras especialidades médicas (39,8\%), em serviços públicos (48\%) e privados de atendimento domiciliar (8,8\%) e por profissionais do Programa Saúde da Família (3,4\%). Os critérios de inclusão foram: ter 60 anos ou mais, estar cuidando de um idoso com dependências físicas e cognitivas, no domicilio, há no mínimo seis meses e ter vínculo de parentesco com o idoso alvo de cuidados. Foram excluídos os cuidadores que apresentaram pontuação abaixo da nota de corte no teste de rastreio para demências CASI-S (Damasceno et al., 2005), em que a nota de corte é de 23 pontos para indivíduos com até 70 anos e de 20 pontos para individuos com idade igual ou acima de 70 anos.

\section{Procedimentos e aspectos éticos}

A coleta foi realizada entre 2014 e 2015, por entrevistadores previamente treinados. 0 estudo "Bem-estar psicológico de idosos que cuidam de outros idosos no contexto da familia" que produziu o banco de dados utilizado para esta investigação foi aprovado pelo Comitê de Ética e Pesquisa da Universidade Estadual de Campinas, aprovado em 6 de outubro de 2014 sob o parecer n. 822.364 e cadastrado na Plataforma Brasil sob o C.A.A.E. 3586 8514.8.0000.5404. Os participantes foram esclarecidos sobre os objetivos, os procedimentos e os aspectos éticos da pesquisa. Todos assinaram um Termo de Consentimento Livre e Esclarecido, antes da coleta de dados.

\section{Variáveis e instrumentos}

Senso de controle. Foi avaliado por meio dos 4 itens do subdomínio "Controle" da Escala CASP19 (Neri et al., 2018). Em sua versão em língua portuguesa, esta escala apresenta uma estrutura composta por dois fatores: "autorrealização e prazer" e "controle e autonomia" e apresenta alta consistência interna para a escala total ( $\alpha$ de Cronbach $=0,850$ ) e para o fator "autorrealização e prazer" ( $\alpha$ de Cronbach $=0,837$ ). O fator "controle e autonomia" apresenta consistência interna intermediária ( $\alpha$ de Cronbach = 0,670), enquanto o subdomínio "controle" apresenta baixa consistência interna ( $\alpha$ de Cronbach = 0,506). Este estudo utilizou os itens deste subdomínio, a saber: "Minha idade me impede de fazer as coisas que gostaria"; "Sinto que não tenho controle sobre o que acontece comigo"; "Sintome livre para planejar o futuro"; e "Sinto-me deixado de lado". Cada item é ancorado por quatro advérbios indicando o grau de concordância dos participantes com a afirmação ( $0=$ nunca, $1=$ às vezes, 2=quase sempre e 3=sempre). Os pontos são somados e podem variar de 0 a 12.

Fragilidade. Foi avaliada por meio de instrumento de autorrelato (Nunes, de Oliveira Duarte, Santos, \& Lebrão, 2015) sobre os seguintes aspectos que compõem o fenótipo de fragilidade descrito por Fried et al. (2001): perda de peso de forma não intencional, nos 3 
meses que antecederam a entrevista; redução da força de preensão; redução da velocidade de caminhada e diminuição de atividades físicas no último ano; e fadiga ao realizar atividades cotidianas na semana anterior à entrevista. Os itens "redução da velocidade de caminhada" e "redução da força de preensão" apresentaram boa consistência interna ( $\alpha$ de Cronbach $=0,77$ e 0,72 , respectivamente), enquanto que os itens "diminuição de atividades físicas", "fadiga ao realizar atividades cotidianas" e "perda de peso de forma não intencional" apresentaram baixa consistência interna ( $\alpha$ de Cronbach = 0,63; 0,37 e 0,31, respectivamente). Apesar da consistência pouco satisfatória de alguns itens, o instrumento tem caráter unidimensional, ou seja, os itens contribuem para o entendimento completo do instrumento, não podendo ser retirados. Pontuavam para fragilidade os idosos que relatassem pelo menos um dos seguintes critérios: perda de mais de 3 kg; redução de força, de velocidade de marcha e de atividades físicas nos últimos 12 meses; e fadiga algumas vezes ou na maior parte do tempo.

Sobrecarga percebida. Utilizou-se a Escala de Sobrecarga de Zarit (Scazufca, 2002), em que o cuidador avalia a sua saúde, o seu bem-estar emocional, as suas relações sociais, a sua vida pessoal e os aspectos financeiros do cuidado. $O$ instrumento apresentou boa consistência interna ( $\alpha$ de Cronbach $=0,870$ ) e é composto por 22 itens de 0 a 4 pontos cada, da menor à maior concordância e escore total de o a 88 pontos.

Religiosidade. Foi avaliada por meio do Índice de Religiosidade de Duke (DUREL) (Taunay et al., 2012), o qual apresentou boa consistência interna ( $\alpha$ de Cronbach $>0,80$ ). O instrumento abrange as dimensões religiosidade organizacional (RO), indicada pela frequência de comparecimento a encontros religiosos; religiosidade não organizacional (RNO), indicada pela frequência de atividades religiosas privadas; e religiosidade intrinseca (RI), ou motivação religiosa para vivenciar a religião no cotidiano. Os dois itens referentes à RO e à RNO são avaliados de 1 a 6 pontos ("mais do que uma vez por semana"; "uma vez por semana"; "duas a três vezes no mês"; "algumas vezes por ano"; "uma vez por ano ou menos"; e "nunca"). Os itens referentes à RI são avaliados de 1 a 5 pontos ("totalmente verdade para mim"; "em geral é verdade"; "não estou certo"; "em geral não é verdade"; e "não é verdade").

Tempo desde o início do cuidado. Este dado foi obtido mediante a pergunta aberta: "há quanto tempo é cuidador?".

Sociodemográficas. Foram coletadas informações a respeito do gênero, da idade dos idosos cuidadores e a presença do vínculo conjugal com o idoso alvo de cuidados.

\section{Análise de dados}

As análises foram feitas por meio do software SAS (Statistical Analysis System) para Windows, versão 9.2 .

A classificação dos valores indicativos dos niveis de senso de controle foi dividida em tercis, seguindo as categorias: "baixo", "intermediário" e "alto". A classificação de fragilidade utilizada foi feita em duas categorias: "robusto" (ausência de pontuação para qualquer um dos cinco critérios) e "em processo de fragilização" (pontuação em um ou mais critérios). Os niveis de sobrecarga foram classificados a partir da distribuição da amostra em tercis, com as categorias "alta", "moderada" e "baixa". As três dimensões de religiosidade, religiosidade organizacional, religiosidade não organizacional e religiosidade intrínseca, têm escore invertido e foram classificadas em alta e baixa de acordo com o valor das medianas, respectivamente: 3, 1 e 4 .

O tempo dispensado ao cuidado de outro idoso foi medido em anos e distribuído em três categorias: menos de dois anos, entre dois e 4,9 anos e maior ou igual a cinco anos. O gênero dos idosos cuidadores foi classificado em masculino e feminino. A idade foi dividida em três faixas: 6064 anos, 65-69 anos e idade maior ou igual a 70 anos. A presença do vínculo conjugal com o idoso alvo de cuidados foi classificada em sim e não.

Foram realizadas análises estatísticas de frequência para as variáveis categóricas e medidas de posição e dispersão para as variáveis 
numéricas. Para comparação das variáveis categóricas, utilizou-se teste o Qui-Quadrado (Conover, 1999; Fleiss, 1981; Tabachnick \& Fidell, 2001). O nivel de significância adotado para todos os testes estatísticos foi de $5 \%(p<0,05)$.

Tendo como embasamento o modelo teórico de Pearlin et al. (1990), construiu-se um Modelo
Inicial de Path Analysis (Figura 1) levando em conta as influências das variáveis independentes (gênero, idade, fragilidade, sobrecarga, religiosidade organizacional, religiosidade não organizacional e religiosidade intrínseca) sobre a variável dependente (senso de controle). A Figura 1 mostra os caminhos testados no Modelo Inicial.

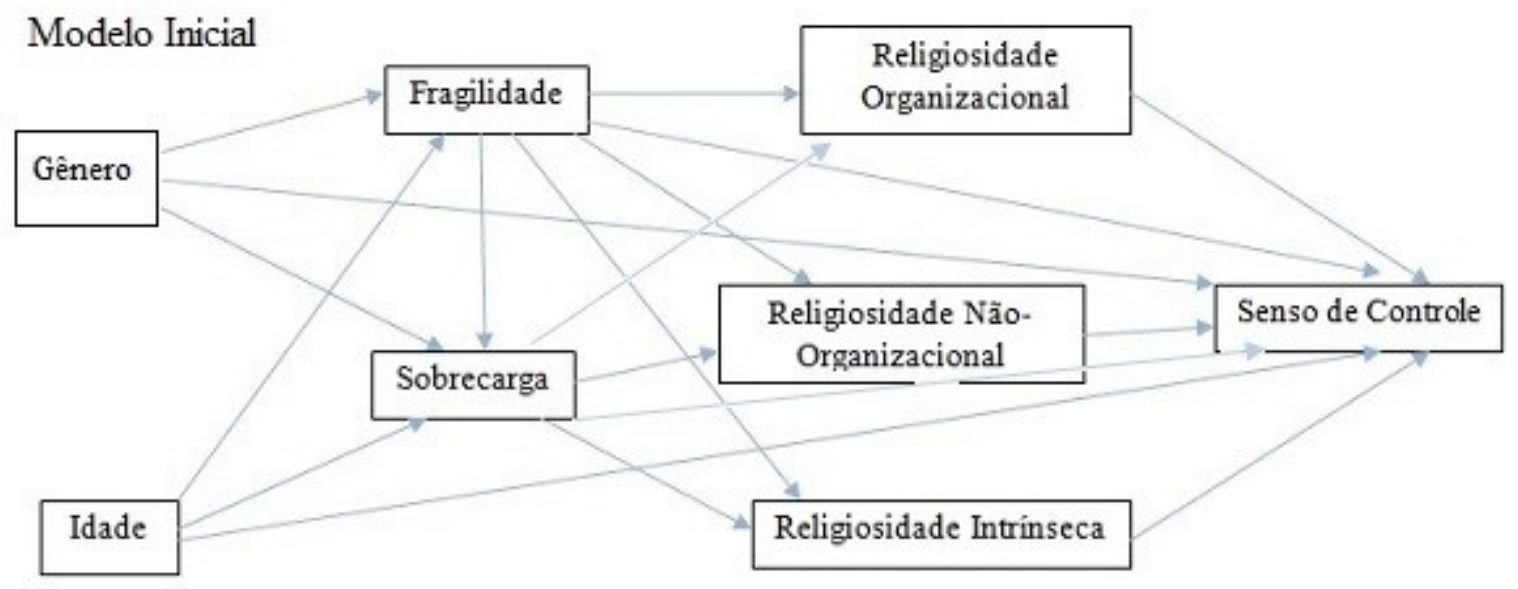

Figura 1 - Modelo inicial testado pela Path Analysis. Campinas, SP, Brasil, 2017.

O teste do modelo proposto foi realizado por meio da análise de equações estruturais via path analysis. Construiu-se um modelo com parâmetros fixos e livres a serem estimados, considerando a adequação dos coeficientes de caminhos frente ao modelo teórico proposto (Anderson \& Gerbing, 1988; Hatcher, 1994). As medidas de ajuste e respectivos critérios de aceitabilidade foram: Teste Qui-Quadrado (c²>0.05); Razão de Qui-Quadrado (c ${ }^{2} / \mathrm{GL}<2$ ); Goodness of Fit Index (GFI $\geq 0.85$ ); GFI Adjusted for Degrees of Freedom (AGFI $\geq 0.80$ ); Standardized Root Mean Square Residual (SRMR $\leq 0.10$ ); Root Mean Square Error of Approximation (RMSEA $\leq 0.08)$; Bentler's Comparative Fit Index (CFI $\geq 0.90)$; e Bentler's \& Bonett's Non-normed Index (NNFI $\geq 0.90$ ).

Para análise da qualidade dos ajustes foram feitos testes de significância para os coeficientes dos caminhos, considerando valores de $t>1.96$ :Teste de Wald para possiveis modificações nos caminhos do modelo inicial e aumento da estatística Qui-Quadrado eTeste de Multiplicadores de Lagrange para verificar a necessidade de criar outros caminhos não considerados no modelo inicial e se há redução ou melhora na estatística Qui-Quadrado, com a sua retirada (Hatcher, 1994).

\section{Resultados}

A amostra foi composta por 148 individuos com idade média de 70 anos ( $\pm 7,1$ ). 36\% dos cuidadores exerciam o cuidado há pelo menos dois e até 4.9 anos e $62 \%$ dos cuidadores apresentavam vínculo conjugal com o idoso alvo de cuidados.

$\mathrm{Na}$ testagem inicial do modelo de path analysis, três medidas apresentaram valores de ajuste aceitáveis: GFI, AGFI e SRMR (0,96, 0,84 e 0,07 , respectivamente). Foi aplicado o Teste de Wald no modelo inicial proposto, o qual indicou necessidade da retirada de 14 caminhos sem valores $\beta$ e t significativos. A realização do Teste de Multiplicadores de Lagrange sugeriu inclusão de dois caminhos (ver Tabela 1). Após única revisão e modificação da análise de caminhos, não houve mais indicações de exclusão ou inclusão de caminhos significativos. Todas as medidas, então, 
apresentaram valores aceitáveis de adequação do ajuste: $C^{2}(0,45), C^{2} / G L(0,98), G F I(0,98), A G F I$ $(0,95)$, SRMR $(0,04)$, RMSEA $(0,0)$, CFI $(1,0)$ e NNFI $(<1,0)$, assim como redução significativa da medida
Qui-Quadrado. A Tabela 1 mostra as estimativas para os coeficientes de caminhos ( $\beta$ ) e para os coeficientes padronizados ( $t$ ) da path analysis no modelo inicial e no modelo final, respectivamente.

Tabela 1 - Estimativa dos coeficientes padronizados dos modelos inicial e final da path analysis. Campinas, SP, Brasil, 2017.

\begin{tabular}{|c|c|c|}
\hline \multicolumn{3}{|c|}{ Modelo Inicial } \\
\hline Caminho de/para & $\beta$ & Valor de $t^{a, b}$ \\
\hline $\mathrm{RO} /$ Senso de Controle & 0,186 & $2,55^{*}$ \\
\hline RNO/Senso de Controle & $-0,025$ & $-0,33$ \\
\hline $\mathrm{RI} /$ Senso de Controle & 0,001 & 0,01 \\
\hline Fragilidade/Senso de Controle & $-0,111$ & $-1,47$ \\
\hline Sobrecarga/Senso de Controle & $-0,356$ & $-4,63^{*}$ \\
\hline Gênero/Senso de Controle & $-0,123$ & $-1,64$ \\
\hline Idade/Senso de Controle & 0,140 & 1,87 \\
\hline Fragilidade/RO & 0,052 & 0,61 \\
\hline Sobrecarga/RO & $-0,027$ & $-0,32$ \\
\hline Fragilidade/RNO & 0,078 & 0,94 \\
\hline Sobrecarga/RNO & $-0,178$ & $-2,15^{*}$ \\
\hline Fragilidade/RI & 0,015 & 0,18 \\
\hline Sobrecarga/RI & $-0,135$ & $-1,61$ \\
\hline Gênero/Fragilidade & $-0,042$ & $-0,49$ \\
\hline Idade/Fragilidade & $-0,081$ & $-0,97$ \\
\hline Fragilidade/Sobrecarga & 0,219 & $2,72^{*}$ \\
\hline Gênero/Sobrecarga & 0,080 & 0,98 \\
\hline Idade/Sobrecarga & 0,051 & 0,61 \\
\hline \multicolumn{3}{|c|}{ Modelo Final } \\
\hline $\mathrm{RO} /$ Senso de Controle & 0,206 & $2,77^{*}$ \\
\hline Sobrecarga/Senso de Controle & $-0,375$ & $-5,05^{*}$ \\
\hline $\mathrm{RNO} / \mathrm{RO}^{\mathrm{c}}$ & 0,180 & $2,28^{*}$ \\
\hline Idade/ROc & 0,209 & $2,63^{*}$ \\
\hline Sobrecarga/RNO & $-0,162$ & $-1,98^{*}$ \\
\hline Fragilidade/Sobrecarga & 0,210 & $2,60^{*}$ \\
\hline
\end{tabular}

Nota. $\beta$ = coeficientes dos caminhos; Valor de $\mathrm{t}=$ coeficientes padronizados; $\mathrm{RO}=$ religiosidade organizacional; $\mathrm{RNO}=$ religiosidade não organizacional; $\mathrm{RI}=$ religiosidade intrinseca.

a Valores de $|t|>1,96$ são considerados significativos para $p<0,05 . R^{2}$ para Senso de Controle: 0,2188; $R^{2}$ para RO: 0,0028; $R^{2}$ para RNO: 0,0320; $R^{2}$ para RI: 0,0175; $R^{2}$ para Fragilidade: 0,0098; $R^{2}$ para Sobrecarga: 0,0547. Residuos (residual terms) E(Senso de Controle): 0,844; E(RO): 0,999; E(RNO): 0,984; E(RI): 0,991; E(Fragilidade): 0,995; E(Sobrecarga): 0,972. bValores de $|t|>1,96$ significativos se $p<0,05 . R^{2}$ para Senso de Controle: 0,1884; $R^{2}$ para $R O$ : 0,0764; $R^{2}$ para RNO: 0,0262; $R^{2}$ para Sobrecarga: 0,0440. Resíduos (residual terms) E(Senso de Controle): 0,901; E(RO): 0,961; E(RNO): 0,987; E(Sobrecarga): 0,978. 'Caminhos incluidos após Teste de Multiplicadores de Lagrange. $* 0,05$. 
O modelo final de path analysis (Figura 2) revelou caminhos entre idade, religiosidade organizacional e senso de controle, assim como entre sobrecarga e senso de controle. Outros caminhos observados foram: fragilidade e sobrecarga, e religiosidade não organizacional e religiosidade organizacional. O modelo final proposto explicou $18,84 \%$ da variabilidade $\left(R^{2}\right)$ do senso de controle, $7,64 \%$ da variabilidade da religiosidade organizacional, 2,62\% da variabilidade da religiosidade não organizacional e $4,4 \%$ da variabilidade da sobrecarga.

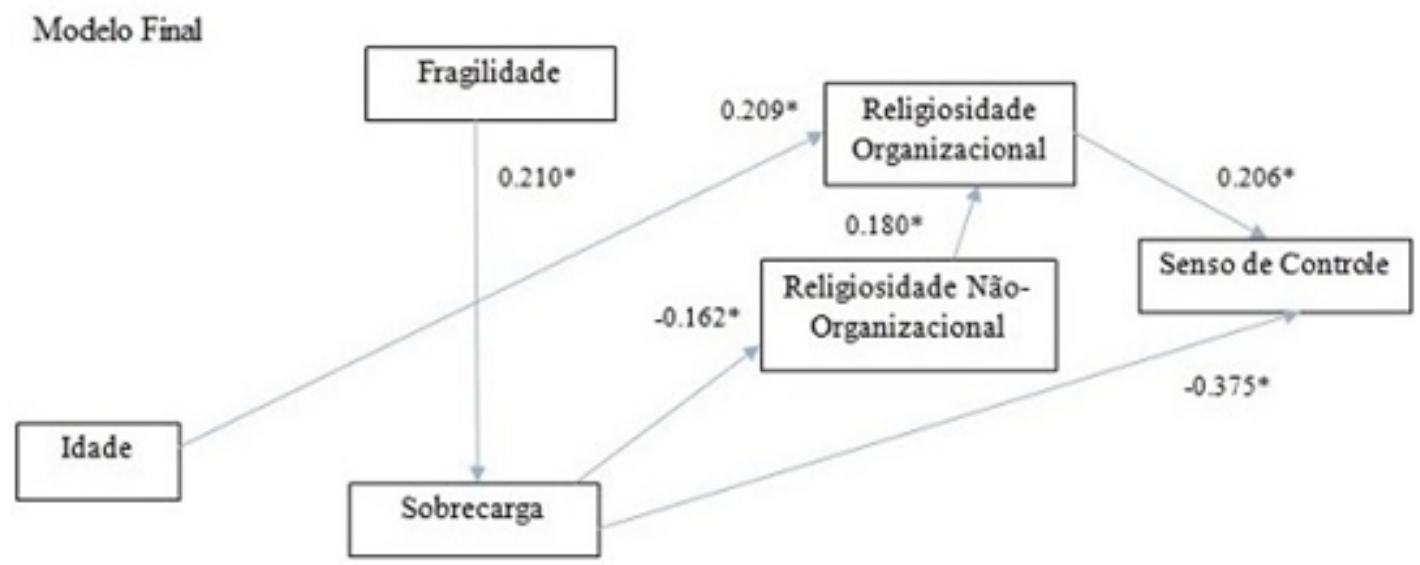

*valores de $\beta$, considerados significativos para $p<0,05$

Na Tabela 2 foi possivel observar diferenças estatisticamente significativas entre senso de controle e sobrecarga percebida, mostrando que os idosos cuidadores com baixa sobrecarga percebida apresentaram alto senso de controle e aqueles com alta sobrecarga percebida apresentaram baixo senso de controle $(p<0,001)$; também houve diferenças significativas entre senso de controle e religiosidade organizacional, em que idosos cuidadores com alta religiosidade organizacional apresentaram alto senso de controle e aqueles com baixa religiosidade organizacional apresentaram senso de controle intermediário e baixo ( $p=0,002)$. A comparação entre as respostas em cada nivel de senso de controle com as demais variáveis não apresentou diferenças estatisticamente significativas.

Tabela 2 - Comparação na proporção de respostas em cada nível de senso de controle. Campinas, SP, Brasil, $2017(N=148)$

Senso de Controle

Total

\begin{tabular}{lcccc}
\hline \multicolumn{1}{c}{ Fragilidade } & Baixo & Intermediário & Alto \\
\hline Robusto & $5(9,09 \%)$ & $11(23,40 \%)$ & $12(26,09 \%)$ & 28 \\
Em processo de fragilização & $50(90,91 \%)$ & $36(76,60 \%)$ & $34(73,91 \%)$ & 120 \\
\hline \multicolumn{5}{c}{ Sobrecarga percebida } \\
\hline Baixa & $10(18,18 \%)$ & $14(29,79 \%)$ & $26(56,52 \%)^{*}$ & 50 \\
Moderada & $16(29,09 \%)$ & $19(40,43 \%)^{*}$ & $13(28,26 \%)$ & 48 \\
Alta & $29(52,73 \%)^{*}$ & $14(29,79 \%)$ & $7(15,22 \%)$ & 50 \\
\hline
\end{tabular}


\begin{tabular}{|l|l|}
\hline $8 / 14$ & Psico, Porto Alegre, v. 51, n. 1, p. 1-14, jan.-mar. $2020 \mid$ e-
\end{tabular}

\begin{tabular}{|c|c|c|c|c|}
\hline \multicolumn{4}{|c|}{ Senso de Controle } & \multirow[t]{2}{*}{ Total } \\
\hline \multicolumn{4}{|c|}{ Religiosidade Organizacional } & \\
\hline Alta & $27(49,09 \%)$ & $19(40,43 \%)$ & $35(76,09 \%)^{* *}$ & 81 \\
\hline Baixa & $28(50,91 \%)^{* *}$ & $28(59,57 \%)^{* *}$ & $11(23,91 \%)$ & 67 \\
\hline \multicolumn{5}{|c|}{ Religiosidade Não Organizacional } \\
\hline Alta & $46(83,64 \%)$ & $39(82,98 \%)$ & $41(89,13 \%)$ & 126 \\
\hline Baixa & $9(16,36 \%)$ & $8(17,02 \%)$ & $5(10,87 \%)$ & 22 \\
\hline \multicolumn{5}{|c|}{ Religiosidade Intrinseca } \\
\hline Alta & $26(47,27 \%)$ & $21(44,68 \%)$ & $25(54,35 \%)$ & 72 \\
\hline Baixa & $29(52,73 \%)$ & $26(55,32 \%)$ & $21(45,65 \%)$ & 76 \\
\hline \multicolumn{5}{|c|}{ Gênero } \\
\hline Masculino & $10(18,18 \%)$ & $10(21,28 \%)$ & $14(30,73 \%)$ & 34 \\
\hline Feminino & $45(81,82 \%)$ & $37(78,72 \%)$ & $32(69.57 \%)$ & 114 \\
\hline \multicolumn{5}{|c|}{ Idade } \\
\hline $60-64$ anos & $11(20,00 \%)$ & $15(31,91 \%)$ & $17(36,96 \%)$ & 43 \\
\hline $65-69$ anos & $13(23,64 \%)$ & $11(23,40 \%)$ & $9(19,57 \%)$ & 33 \\
\hline$\geq 70$ anos & $31(56,36 \%)$ & $21(44,68 \%)$ & $20(43,48 \%)$ & 72 \\
\hline Total & 55 & 47 & 46 & 148 \\
\hline
\end{tabular}

Nota. ${ }^{*} p<0,001{ }^{* *} p=0,002$

Conforme a Tabela 3 verificou-se que a maioria da amostra apresentou senso de controle intermediário e alto. O processo de fragilização estava presente na maior parte da amostra. A maioria da amostra apresentou sobrecarga percebida moderada e alta, assim como alta religiosidade organizacional, alta religiosidade não organizacional e baixa religiosidade intrinseca. Além disso, a amostra foi composta majoritariamente por mulheres (77\%).

Tabela 3 - Pontuações em senso de controle, fragilidade, sobrecarga percebida, religiosidade, gênero e idade de idosos cuidadores. Campinas, SP, Brasil, 2017 ( $N=148)$

\begin{tabular}{lcc}
\hline & $\boldsymbol{N}(\%)$ & $\boldsymbol{M}(\boldsymbol{D P})$ \\
\hline Senso de Controle & & $8,93(2,53)$ \\
$\leq 8$ pontos (baixo) & $55(37,16 \%)$ \\
9-10 pontos (intermediário) & $47(31,76 \%)$ \\
11-12 pontos (alto) & $46(31,08 \%)$ \\
Fragilidade & \\
Robusto & \\
Em processo de fragilização & $28(18,92 \%)$ \\
\end{tabular}




\begin{tabular}{|c|c|c|}
\hline & $N(\%)$ & $M(D P)$ \\
\hline Sobrecarga percebida & & $26,14(13,51)$ \\
\hline 0-19 pontos (baixa) & $50(33.78 \%)$ & \\
\hline 20-27 pontos (moderada) & $48(32,43 \%)$ & \\
\hline$\geq 28$ pontos (alta) & $50(33,78 \%)$ & \\
\hline Religiosidade Organizacional & & $3,61(1,89)$ \\
\hline 1 a 3 pontos (alta) & $81(54,73 \%)$ & \\
\hline 4 a 6 pontos (baixa) & $67(45,27 \%)$ & \\
\hline \multicolumn{3}{|c|}{ Religiosidade Não Organizacional } \\
\hline 1 ponto (alta) & $126(85,14 \%)$ & \\
\hline 2 a 6 pontos (baixa) & $22(14,86 \%)$ & $1,40(1,19)$ \\
\hline Religiosidade Intrínseca & & $4,36(2,08)$ \\
\hline 3 pontos (alta) & $72(48,65 \%)$ & \\
\hline 4 a 15 pontos (baixa) & $76(51,35 \%)$ & \\
\hline \multicolumn{3}{|l|}{ Gênero } \\
\hline Masculino & $34(22,97 \%)$ & \\
\hline Feminino & $114(77,03 \%)$ & \\
\hline Idade & & $69,73(7,07)$ \\
\hline $60-64$ anos & $43(29,05 \%)$ & \\
\hline $65-69$ anos & $33(22,30 \%)$ & \\
\hline$\geq 70$ anos & $72(48,65 \%)$ & \\
\hline
\end{tabular}

\section{Discussão}

O presente estudo teve como objetivo principal testar um modelo de path analysis e verificar se houve associações estatisticamente significativas do senso de controle de idosos cuidadores de idosos no contexto familiar com as seguintes variáveis: fragilidade, sobrecarga percebida, religiosidade, gênero e idade dos idosos cuidadores. Por meio da path analysis, encontrou-se que o senso de controle esteve associado à idade, por meio da religiosidade organizacional, e à sobrecarga. Além disso, também se associaram de forma significativa as variáveis fragilidade e sobrecarga, e as variáveis religiosidade não organizacional e religiosidade organizacional.

Este estudo também teve como objetivo a comparação na proporção de respostas dos idosos cuidadores nos niveis de senso de controle segundo niveis de fragilidade, de sobrecarga percebida, de religiosidade, gênero e idade da amostra. A partir do teste qui-quadrado, observou-se que os idosos cuidadores com baixa sobrecarga apresentaram alto senso de controle. Ademais, verificou-se que os idosos cuidadores com alta religiosidade organizacional apresentaram alto senso de controle.

As análises descritivas encontraram que a maioria da amostra apresentou senso de controle intermediário e alto e oitenta por cento estava em processo de fragilização. Ainda foi encontrado que a maioria da amostra apresentou sobrecarga moderada e alta, alta religiosidade organizacional, alta religiosidade não organizacional e baixa religiosidade intrinseca. A amostra foi composta majoritariamente pelo gênero feminino e com idade média de 70 anos.

O modelo de path analysis, apresentado neste estudo, revelou que o aumento da idade se associou ao aumento do senso de 
controle por meio do aumento da religiosidade organizacional. A associação distal entre idade e senso de controle, mediada por religiosidade organizacional, sugere que os idosos encontram apoio na religião para lidar com perdas comuns ao envelhecimento, como os declínios em saúde (Bengtson, Silverstein, Putney, \& Harris, 2015). Nesse sentido, os idosos cuidadores da presente pesquisa parecem valorizar a prática religiosa pública e mesmo com idade avançada conseguem frequentar encontros religiosos, assim como encontrado no estudo de Koenig et al. (2016). Levando em conta a associação entre religiosidade organizacional e senso de controle, podemos cogitar que, por meio da prática pública da religião, o idoso cuidador vivencie a crença do "controle mediado por Deus", descrito por Krause (2007), em que ele acredita que compartilhar o controle com Deus pode ajudar a superar circunstâncias estressantes atuais em sua vida, dentre elas o cuidado a outro idoso. Esta crença fomenta o senso de controle do idoso cuidador e auxilia a manutenção do bem-estar psicológico (Krause \& Hayward, 2014). A rede de apoio social presente nas instituições religiosas também impulsiona o senso de controle mediado por Deus (Krause, 2007) e pode oferecer a possibilidade de aceitação das dificuldades do cuidado, assim como promover senso de significado e de propósito frente ao exercício do cuidado (Koenig et al., 2016).

No presente estudo, as variáveis sobrecarga e senso de controle se associaram de forma inversamente proporcional, em que o aumento da percepção de sobrecarga se associou à diminuição do senso de controle e a diminuição da percepção de sobrecarga se associou ao aumento do senso de controle. Esse dado sugere que, nessa amostra, o aumento de sobrecarga decorrente do exercício do cuidado prejudica a capacidade do idoso cuidador de lidar de forma ativa e adequada com o ambiente, refletindose em prejuizos ao senso de controle e ao seu bem-estar psicológico. A literatura que investiga as relações entre sobrecarga do cuidador e senso de controle ainda é escassa, porém
Rafnsson e colaboradores (2015) encontraram que cônjuges tinham sobrecarga elevada, por conta de demandas do cuidado, tais como auxilio em atividades trabalhosas e cansativas como dar banho, ajudar a se levantar e a se trocar, associadas à diminuição do senso de controle em um periodo de dois anos. A literatura aponta que cuidadores idosos experimentam algum indice de sobrecarga (Potier et al., 2018), principalmente quando são mulheres cuidando do cônjuge (Swinkels, Tilburg, Verbakel \& Broese van Groenou, 2017). Assim, mais pesquisas sobre a relação entre sobrecarga e senso de controle são necessárias para compreender possiveis consequências negativas ao bem-estar psicológico do idoso cuidador.

Nessa amostra, houve associação das variáveis fragilidade e sobrecarga, em que idosos em processo de fragilização apresentaram sobrecarga mais alta do que os robustos. Possivelmente as tarefas de cuidar geram estresse físico e psicológico ao idoso cuidador que apresenta algum nivel de fragilidade, o que, por sua vez, intensifica o senso de sobrecarga, assim como encontrado por Sun (2014), ainda que os idosos da presente amostra tivessem uma rede de apoio formal estruturada, demonstrada pelo uso de serviços especializados em geriatria. Brigola et al. (2017) investigaram 85 idosos cuidadores moradores de áreas rurais de São Carlos-SP e referiram que essa população pode apresentar maiores indices de fragilidade e sobrecarga por conta da restrição ao acesso à rede de apoio formal no cuidado à própria saúde e no cuidado a outro idoso. Os autores encontraram que aqueles idosos clinicamente frágeis e com sobrecarga severa têm piores desempenhos em teste de cognição global, influenciando a qualidade de vida do idoso cuidador e do cuidado prestado, podendo aumentar ainda mais a sobrecarga.

No que tange à associação entre as variáveis religiosidade não organizacional e religiosidade organizacional, em que o aumento da primeira está associado ao aumento da segunda, podemos cogitar que a prática religiosa privada para o idoso cuidador de outro idoso é um momento de 
descanso no cotidiano da prestação de cuidados, promovendo melhor adaptação ao papel de cuidador e senso de propósito diante das tarefas do cuidado (Koenig et al., 2016). Porém, o idoso cuidador pode sentir necessidade de maior apoio religioso e aumentar a sua participação em encontros religiosos. É bem estabelecido na literatura que a religiosidade organizacional tende a aumentar entre idosos jovens e a diminuir na velhice avançada, uma vez que no primeiro grupo a diminuição de responsabilidades familiares e no trabalho faz com que estes idosos tenham mais tempo livre para frequentar encontros religiosos. No segundo grupo, por outro lado, a ida constante a encontros religiosos pode ser dificultada pela diminuição da capacidade física funcional e da saúde geral (Bengtson et al., 2015; Hayward \& Krause, 2013). Na velhice, questões espirituais parecem estar mais proeminentes, havendo maior consciência da finitude e senso de completude, e sendo importante, inclusive, o papel da religiosidade no ajustamento psicológico a várias perdas relacionadas ao envelhecimento, inclusive na diminuição da plena capacidade funcional (Bengtson et al., 2015; Hayward \& Krause, 2013). Além disso, a frequência constante em encontros religiosos, nessa faixa etária, pode trazer bem-estar, felicidade, emoções positivas, esperança e otimismo, suporte social, assim como reduzir o sofrimento que o contexto do cuidado pode causar dentre idosos que cuidam de outros idosos (Koenig, 2012; Koenig et al., 2016). No cenário do cuidado, porém, o achado acima mencionado aponta a necessidade de novas pesquisas que investiguem a relação entre religiosidade não organizacional e religiosidade organizacional para melhor compreensão de aspectos e mecanismos desta relação entre idosos cuidadores de idosos.

Os dados do qui-quadrado corroboram os achados da path analysis e mostram a importância da diminuição da sobrecarga percebida para a manutenção da crença de capacidade de atuar sobre o ambiente de modo adequado e adaptado dentre cuidadores idosos de outros idosos. De forma contrária, maior sobrecarga percebida em decorrência de prestar cuidado a outro idoso prejudicou o senso de controle dos idosos cuidadores, assim como encontrado no estudo de Rafnsson et al. (2015), reforçando que, nessa amostra, a avaliação negativa que o idoso cuidador faz das modificações em vários setores da vida após o início do cuidado parece importante na diminuição da crença na própria capacidade de controlar ativamente elementos do ambiente do cuidado. Outro achado significativo do teste qui-quadrado revelou que cuidadores idosos que referiram alta religiosidade organizacional pontuaram alto em controle percebido, enquanto aqueles idosos que referiram baixa religiosidade organizacional apresentaram niveis intermediário e baixo de senso de controle. Esse dado corrobora a literatura que demonstrou que a prática religiosa pública pode aumentar o senso de controle (Krause, 2007) e mostra a relevância da religiosidade organizacional na manutenção e no aumento da crença de ser capaz de exercer controle ativo sobre o ambiente, podendo ser considerada um fator de proteção ao bem-estar psicológico do cuidador idoso (Krause \& Hayward, 2014).

A amostra da presente pesquisa foi, em sua maioria, composta por mulheres, assim como nos estudos de Brigola et al. (2017), Santos-Orlandi et al. (2017a), Santos-Orlandi et al. (2017b) e Potier et al. (2018). Swinkels et al. (2017) referem que a prestação de cuidados por mulheres configura um perfil tradicional de cuidadores, visto que o cuidado ainda é visto como atividade essencialmente feminina.

A alta porcentagem da amostra em processo de fragilização (81\%) difere dos achados em outros estudos com cuidadores idosos brasileiros (Brigola et al., 2017; Santos-Orlandi et al., 2017a; SantosOrlandi et al., 2017b). Esse achado demonstra que a presente amostra pode ter suas reservas fisiológicas ainda mais diminuidas, bem como o funcionamento físico e motor possivelmente mais limitado, com maior risco de agravos ao longo dos anos (Potier et al., 2018) do que outras amostras brasileiras com menor fragilidade.

A sobrecarga foi referida como moderada e alta pelos idosos cuidadores, corroborando os achados 
de Potier et al. (2018) mas diferindo de Brigola et al. (2017), o que pode se dever aos diferentes métodos de classificação da pontuação das amostras na Escala de Sobrecarga de Zarit. Esse dado demonstra que os idosos cuidadores dessa amostra avaliaram que a situação do cuidado a outro idoso modificou suas vidas de forma negativa.

Os altos índices de religiosidade organizacional e de religiosidade não organizacional encontrados confirmam uma especificidade da coorte idosa, referida por Bengtson et al. (2015), mostrando que as práticas religiosas são altamente difundidas entre idosos brasileiros (Instituto Brasileiro de Geografia e Estatistica [IBGE], 2010). Ratificam ainda que a religiosidade pode ser fonte de suporte social, auxiliando na diminuição de sentimentos de isolamento e solidão, os quais costumam ser referidos por cuidadores, e na manutenção do bem-estar psicológico de idosos cuidadores (Koenig, 2012; Koenig et al., 2016).

Portanto, os achados do presente estudo elucidam que a idade mais avançada, maior religiosidade organizacional e menos sobrecarga percebida são fatores que contribuem para o fortalecimento do senso de controle de idosos cuidadores que cuidam de outros idosos no contexto domiciliar. A presente pesquisa apresentou como limitação o fato de a amostra ser de conveniência, o que faz com que os dados encontrados tenham menor generalidade para a população geral de idosos que cuidam de outros idosos no contexto familiar.

\section{Considerações finais}

A manutenção do senso de controle é um elemento-chave no bem-estar psicológico e na qualidade de vida subjetiva na velhice. Dessa forma mostra-se relevante um melhor entendimento da percepção/avaliação que idosos cuidadores de outros idosos têm/fazem dos elementos do cuidado e dos recursos pessoais que podem ser utilizados para lidar com esse cenário.

Futuros estudos deverão tentar compreender mais profundamente a forma como se estabelece a relação entre o senso de controle, a idade, a religiosidade e a sobrecarga. Assim como deverão investir na compreensão dessa relação em termos longitudinais, de forma a fomentar ações que levem em conta as especificidades próprias do envelhecer de idosos cuidadores.

\section{Referências}

Anderson, J. C. \& Gerbing, D. W. (1988). Structural equation modeling in practice: A review and recommended two-step approach. Psychological Bulletin, 103(3), 411-423. https://doi.org/10.1037/00332909.103.3.411

Bengtson, V. L., Silverstein, M., Putney, N. M., \& Harris, S. C. (2015). Does religiousness increase with age? Age changes and generational differences over 35 years. Journal For The Scientific Study Of Religion, 54(2), 363-379. https://doi.org/10.1111/jssr.12183

Bianchi, M. (2015). Indicadores de sobrecarga, estratégias de enfrentamento e sintomas depressivos em idosos que cuidam de outros idosos (Dissertação de Mestrado). Universidade Estadual de Campinas, Campinas, Brasil. Recuperado de http://repositorio. unicamp.br/ jspui/ handle/REPOSIP/313052 (DOI INEXISTENTE)

Brigola, A. G., Luchesi, B. M., Alexandre T. S., Inouye, K., Mioshi, E., \& Pavarini, S. C. I. (2017). High burden and frailty: association with poor cognitive performance in older caregivers living in rural areas. Trends In Psychiatry And Psychotherapy, 39(4), 257-263. https://doi.org/10.1590/2237-6089-2016-0085

Conover, W. J. (1999). Practical Nonparametric Statistics. New York: John Wiley \& Sons. (DOI INEXISTENTE)

Damasceno, A., Delicio, A. M., Mazo, D. F., Zullo, J. F., Scherer, P., Ng, R. T., \& Damasceno, B. P. (2005). Validation of the Brazilian version of mini-test CASI-S. Arquivos De Neuro-Psiquiatria, 63(2B), 416-421. https:// doi.org/10.1590/S0004-282X2005000300010

Dos Santos, R. L. \& Virtuoso, J. S., Jr. (2008). Confiabilidade da versão brasileira da Escala de Atividades Instrumentais de Vida Diária. Revista Brasileira em Promoção da Saúde, 21(4), 290-296. (DOI INEXISTENTE)

Fleiss, J. L. (1981). Statistical Methods for Rates and Proportions. New York: John Wiley \& Sons. (DOI INEXISTENTE)

Fried, L. P., Tangen, C. M., Walston, J., Newman, A. B. Hirsch, C., Gottdiener, J., Seeman, T., Tracy, R., Kop, W. J., Burke, G., \& McBurnie, M. A. (2001). Frailty in older adults: evidence for a phenotype. Journal of Gerontology: Medical Sciences, 56(3), M146-M157. https://doi.org/10.1093/gerona/56.3.M146

Hatcher, L. (1994). A step-by-step approach to using the SAS System for factor analysis and structural equation modeling. Cary, NC: SAS Institute Inc., 588p. (DOI INEXISTENTE) 
Hayward, R. D. \& Krause, N. (2013). Patterns of change in religious service attendance across the life course: Evidence from a 34-year longitudinal study. Social Science Research, 42(6), 1480-1489. https://doi org/10.1016/j.ssresearch.2013.06.010

Hyde, M., Wiggins, R. D., Higgs, P., \& Blane, D. B. (2003). A measure of quality of life in early old age: the theory, development and properties of a needs satisfaction model (CASP-19). Aging \& Mental Health, 7(3), 186-194. https://doi.org/10.1080/1360786031000101157

Instituto Brasileiro de Geografia e Estatística (2010). Censo Demográfico 2010: Características gerais da população, religião e pessoas com deficiência. Recuperado de http://www.ibge.gov.br/home/estatistica/populacao/censo2010/caracteristicas_religiao_deficiencia/caracteristicas_religiao_deficiencia_tab_xls.shtm (DOI INEXISTENTE)

Jones, A. J., Kuijer, R. G., Livingston, L., Myall, D., Horne, K., MacAskill, M., ... \& Dalrymple-Alford, J. C. (2017). Caregiver burden is increased in Parkinson's disease with mild cognitive impairment (PD-MCl). Translational Neurodegeneration, 6(17), 1-9. https:// doi.org/10.1186/s40035-017-0085-5

Koenig, H. G. (2017). Religion and spirituality in gerontology. In: M. Balboni \& J. Peteet (Org.), Spirituality and religion within the culture of medicine: from evidence to practice (pp. 109-127). New York: Oxford University Press. (DOI INEXISTENTE)

Koenig, H. G. (2012). Religion, spirituality, and health: the research and clinical implications. International Scholarly Research Network Psychiatry, 2012. https:// doi.org/10.5402/2012/278730

Koenig, H. G., Nelson, B., Show, S. F., Saxena, S., \& Cohen, H. J. (2016). Religious involvement and adaptation in female caregivers. Journal Of American Geriatrics Society, 64(3), 578-583. https://doi org/10.1111/jgs.13929

Krause, N. \& Hayward, R. D. (2014). Religion, finding interests in life, and change in self-esteem during late life. Research On Aging, 36(3), 364-381. https:// doi.org/10.1177/0164027513496437

Krause, N. (2007). Social involvement in religious institutions and God-mediated control beliefs: a longitudinal investigation. Journal For The Scientific Study Of Religion, 46(4), 519-537. https://doi.org/10.1111/j. 1468-5906.2007.00375.x

Lino, V. T. S., Pereira, S. R. M., Camacho, L. A. B., Ribeiro Filho, S. T., \& Buksman, S. (2008). Adaptação transcultural da escala de independência em atividades de vida diária (Escala de Katz). Cadernos de Saúde Pública, 24(1), 103-112. https://doi.org/10.1590/ S0102-311X2008000100010

Montaño, M. B. M. M., \& Ramos, L. R. (2005). Validade da versão em português da clinical dementia rating. Revista de Saúde Pública, 39(6), 912-917. https://doi. org/10.1590/So034-89102005000600007
Neri, A. L., Borim, F. S. A., Batistoni, S. S. T., Cachioni, M., Rabelo, D. F., Fontes, A. P., \& Yassuda, M. S. Nova validação semântico-cultural e estudo psicométrico da CASP-19 em adultos e idosos brasileiros. (2018). Cadernos de Saúde Pública, 34(10), e:00181417. https://doi.org/10.1590/0102-311X00181417

Nunes, D. P., de Oliveira Duarte, Y. A., Santos, J. L. F., \& Lebrão, M. L. (2015). Rastreamento de fragilidade em idosos por instrumento autorreferido. Revista de Saúde Pública, 49, 1-9. https://doi.org/10.1590/ So034-8910.2015049005516

Oldenkamp, M., Hagedoorn, M., Wittek, R., Stolk, R., \& Smidt, N. (2017). The impact of older person's frailty on the care-related quality of life of their informal caregiver over time: results from the TOPIC-MDS project. Quality Of Life Research,26(10), 2705-2716. https://doi.org/10.1007/s11136-017-1606-5

Pearlin, L. I., Mullan, J. T., Semple, S. J., \& Skaff, M. M. (1990). Caregiving and the stress process: an overview of concepts and their measures. The Gerontologist, 30(5), 583-591. https://doi.org/10.1093/ geront/30.5.583

Pearlin, L. I. (2009). The life course and the stress process: some conceptual comparisons. Journal of Gerontology: Social Sciences, 65B(2), 207-215. https:// doi.org/10.1093/geronb/gbp106

Potier, F., Degryse, J. M., Bihin, B., Debacq-Chainiaux, F., Charlet-Renard, C., Martens, H., \& de Saint-Hubert, M. (2018). Health and frailty among older spousal caregivers: an observational cohort study in Belgium. BMC Geriatrics, 18(1), 291. https://doi.org/10.1186/ S12877-018-0980-3

Rafnsson, S. B., Shankar, A., \& Steptoe, A. (2015). Informal caregiving transitions, subjective well-being and depressed mood: findings from the English Longitudinal Study of Ageing. Aging \& Mental Health, o(0), 1-9. https://doi.org/10.1080/13607863.2015.1088510

Santos-Orlandi, A. A., Brito, T. R. P., Ottaviani, A. C., Rossetti, E. S., Zazzetta, M. S., Gratão, A. C. M., Orlandi, F. S., \& Pavarini, S. C. I. (2017a). Perfil de idosos que cuidam de outros idosos em contexto de alta vulnerabilidade social. Escola Anna Nery, 21(1), e20170013. https://doi.org/10.5935/1414-8145.20170013

Santos-Orlandi, A. A., Brito, T. R. P., Ottaviani, A. C., Rossetti, E. S., Zazzetta, M. S., \& Pavarini, S.C. I. (2017b). Elderly who take care of elderly: a study on the Frailty Syndrome. Revista Brasileira De Enfermagem, 70(7), 822-829. https://doi.org/10.1590/00347167-2016-0474

Scazufca, M. (2002). Brazilian version of the Burden Interview scale for the assessment of burden of care in carers of people with mental illnesses. Revista Brasileira De Psiquiatria, 24(1), 12-17. https://doi. org/10.1590/S1516-44462002000100006

Sexton, E., King-Kallimaris, B. L., Layte, R., \& Hickey, A. (2015). CASP-19 special section: how does chronic disease status affect CASP quality of life at older ages? Examining the WHO ICF disability domains as mediators of this relationship. Aging \& Mental Health, 19(7), 622-633. https://doi.org/10.1080/13607863.2014.955457 
Sun, F. (2014) Caregiving stress and coping: a thematic analysis of chinese family caregivers of persons with dementia. Dementia, 16(6), 803-818. https://doi. org/10.1177/1471301213485593

Swinkels, J., Tilburg, T. V., Verbakel, E., \& Broese van Groenou, M. (2017). Explaining the Gender Gap in the Caregiving Burden of Partner Caregivers. Journals of Gerontology: Social Sciences, 74(2), 309-317. https:// doi.org/10.1093/geronb/gbx036

Tabachnick, B. G. \& Fidell, L. S. (2001). Using multivariate statistics. Boston: Allyn and Bacon. (DOI INEXISTENTE)

Taunay, T. C. D., Gondim, F. D. A. A., Macêdo, D. S., Moreira-Almeida, A., Gurgel, L. D. A., Andrade, L. M. S., \& Carvalho, A. F. (2012). Validação da versão brasileira da escala de religiosidade de Duke (DUREL). Archives Of Clinical Psychiatry (São Paulo), 39(4), 130-5. https://doi.org/10.1590/S0101-60832012000400003

\section{Endereço para correspondência:}

Larissa Centofanti Lemos (Rua Tessália Vieira de Camargo, 126, Campinas, São Paulo, 13083-970; (19)35217408/(11)97662-2214; larissacentofanti@gmail.com).

\section{Autor I}

Nome: Larissa Centofanti Lemos.

Titulação Acadêmica: Mestrado em Gerontologia (UNICAMP).

Afiliação Institucional: Universidade Estadual de Campinas (UNICAMP).

\section{Autor II}

Nome: Samila Sathler Tavares Batistoni.

Titulação Acadêmica: Doutorado em Educação - UNICAMP.

Afiliação Institucional: Universidade de São Paulo (USP).

\section{Autor III}

Nome: Meire Cachioni.

Titulação Acadêmica: Doutorado em Educação - UNICAMP.

Afiliação Institucional: Universidade de São Paulo (USP).

\section{Autor IV}

Nome: Anita Liberalesso Neri.

Titulação Acadêmica: Doutorado em Psicologia Escolar e do Desenvolvimento Humano (USP).

Afiliação Institucional: Universidade Estadual de Campinas (UNICAMP). 